\title{
Research and Application of WebGIS in Coal Mine Information Management System
}

\author{
Xiucai Guo ${ }^{1, a}$, Renjun Wang ${ }^{1, b}$ and Zhen $\mathrm{Wu}^{1, \mathrm{c}}$ \\ ${ }^{1}$ Xi'an University of Science and Technology, Xi'an, 710054, China \\ aguoxiucai@163.com, b664740245@qq.com, '870082058@qq.com
}

Keywords: WebGIS, Coal mine, Dispatching drawing, C/S+B/S, Spatial database

Abstract. The efficient coal mine dispatching graphics digital system not only t can realize the unified management of information resources, but also can make efficient and accurate policy decision through the feedback of information, which effectively solve the problem of information of insular islands in coal mine. Coal mine information dispatching management system is designed based on GIS technology, WEB technology, database technology and monitoring technology to realize digital management of coal mine information dispatching and management based on $\mathrm{C} / \mathrm{S}+\mathrm{B} / \mathrm{S}$ mixed mode structure. The database system and the function of the data engine is introduced and the design scheme of WebGIS based on Ajax and ASP.NET is given in the paper. Through real-time chart information, the real situation of each coal mine production can be reflected directly. The system can realize the improvement and optimization of the system and improve the coal mine of level in the management, the safety and the automation.

\section{Introduction}

As the center of the whole coal mine production, the Information processing of coal production scheduling system become very important. The system can improve the management level of coal mine, not only to realize the unified management of information resources, but also through the feedback of information to make efficient and accurate policy decision. Therefore, optimizing and improving the function of coal mine production scheduling is very important[1].

With the rapid development of Geographic Information System (GIS), Web technology, database technology and computer technology, the dynamic graphics processing and service system is established based on integrating together the spatial data engine (SDE), real-time data engine and monitoring system[2]. The system can realize the real-time acquisition and display of data, unified management of thematic graphics and visualization analysis of production information. Therefore, it will improve the coal mine of level in the management, the safety and the automation. Mine information management information management system including Network architecture of client/server $(\mathrm{C} / \mathrm{S})$ and browser/server $(\mathrm{B} / \mathrm{S})$ can realize visual information management of image, production information, equipment and Mine environmental parameters. Using Visual Basic6.0 as the software platform, visual graphic digital system is developed based on software including MapInfo and SQL Server. The design of WebGIS based on Ajax,ASP.NET and real-time access to the database, achieve the scheduling of graphics browsing, query and other functions of the improvement and optimization.

\section{Summary of WebGIS}

The World Wide Web Geographic Information System (WebGIS) is the computer information system of geographic information adapting communication and application protocols to Store, process, analyze and display system information, which is a new technology based on Web[3]. The basic idea of WebGIS is to provide geographic information on the Internet, then deal with the graph with the computer and let users access to a geographic information system in the data and functional services with browsing through the browser. According, it has a strong user operating function. Technically, 
WebGIS is the integration of GIS technology and component technology, Interoperability Technology and distributed technology.

At present, there have been a variety of different technical methods for the construction and development of WebGIS, including Common Gateway Interface (CGI), Server API, Plug-in, Ajax asynchronous component technology and Java programming technology.

\section{Design of System}

Network of System. Information management system of coal mine information management includes graphics processing function and graph browsing function, which adopt the network structure model of $\mathrm{B} / \mathrm{S}+\mathrm{C} / \mathrm{S}$. The network structure of the system is shown in Fig.1.

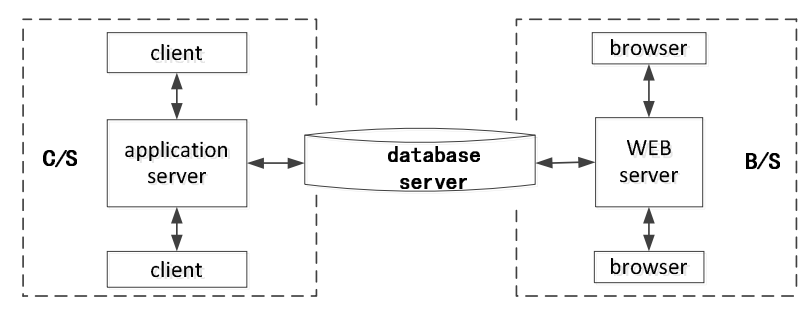

Fig. 1 The network structure of the system

Overall Design of System. The system is the visual information management system based on composition of Visual Basic6.0, MapInfo and SQL Server. The whole is divided into three parts: data part, graphic part and interface part[4]. The overall design structure of the system is shown in Fig. 2.

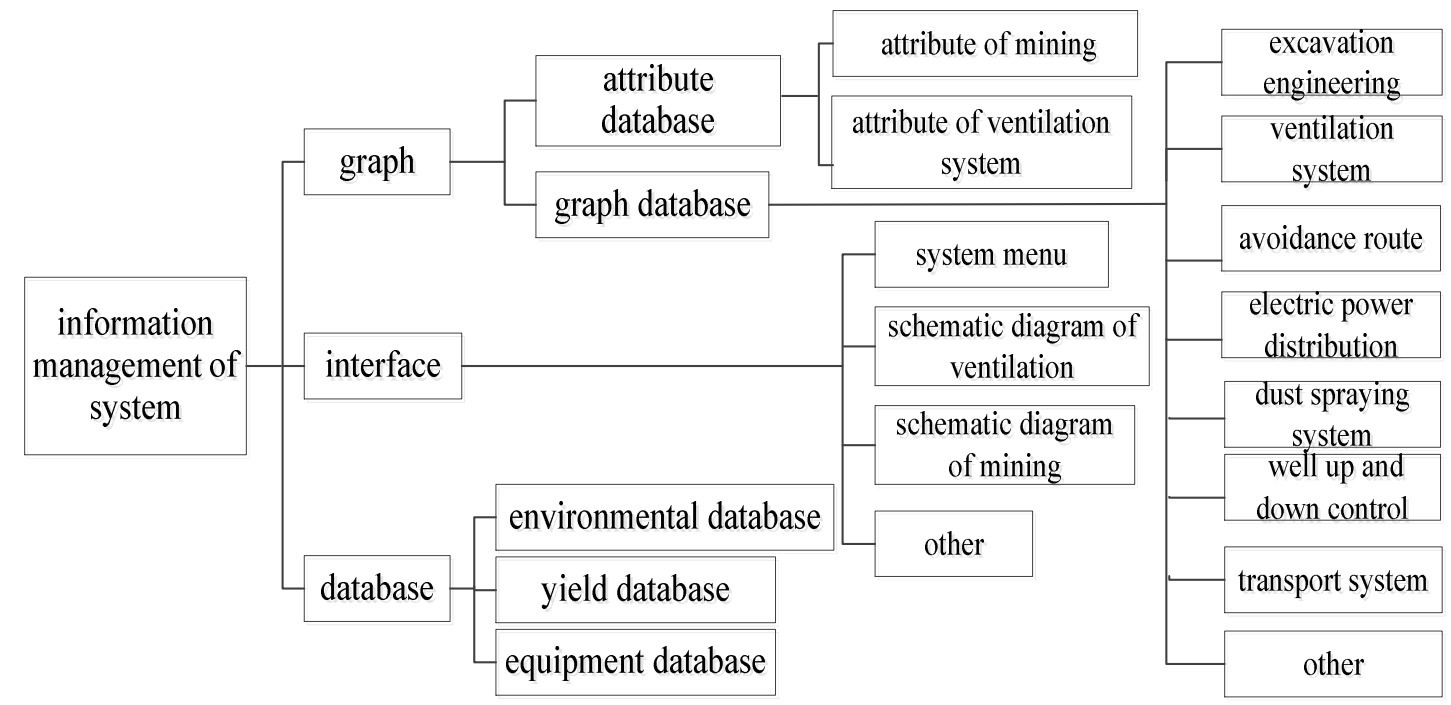

Fig. 2 The overall design structure of the system

The data part take the device database as template to achieve structured design, which includes data entry, data retained for future reference, the display of data graph and other functions. The graphic part includes two parts, the graphic module and the attribute module, which also includes the data of desktop graphics and the various attribute information in the graph. The interface of system adopts the design visual function based on simple, clear and easy requirements.

\section{Function realization of database system}

The system data is divided into two parts: the basic data of geographic information and the data of coal mine dispatching and management. The data in GIS system includes spatial data and attribute 
data[5]. Because the spatial data and attribute data are interrelated rather than isolated, the system uses the whole relational database management. Database composition and logical relations are shown in Fig. 3.

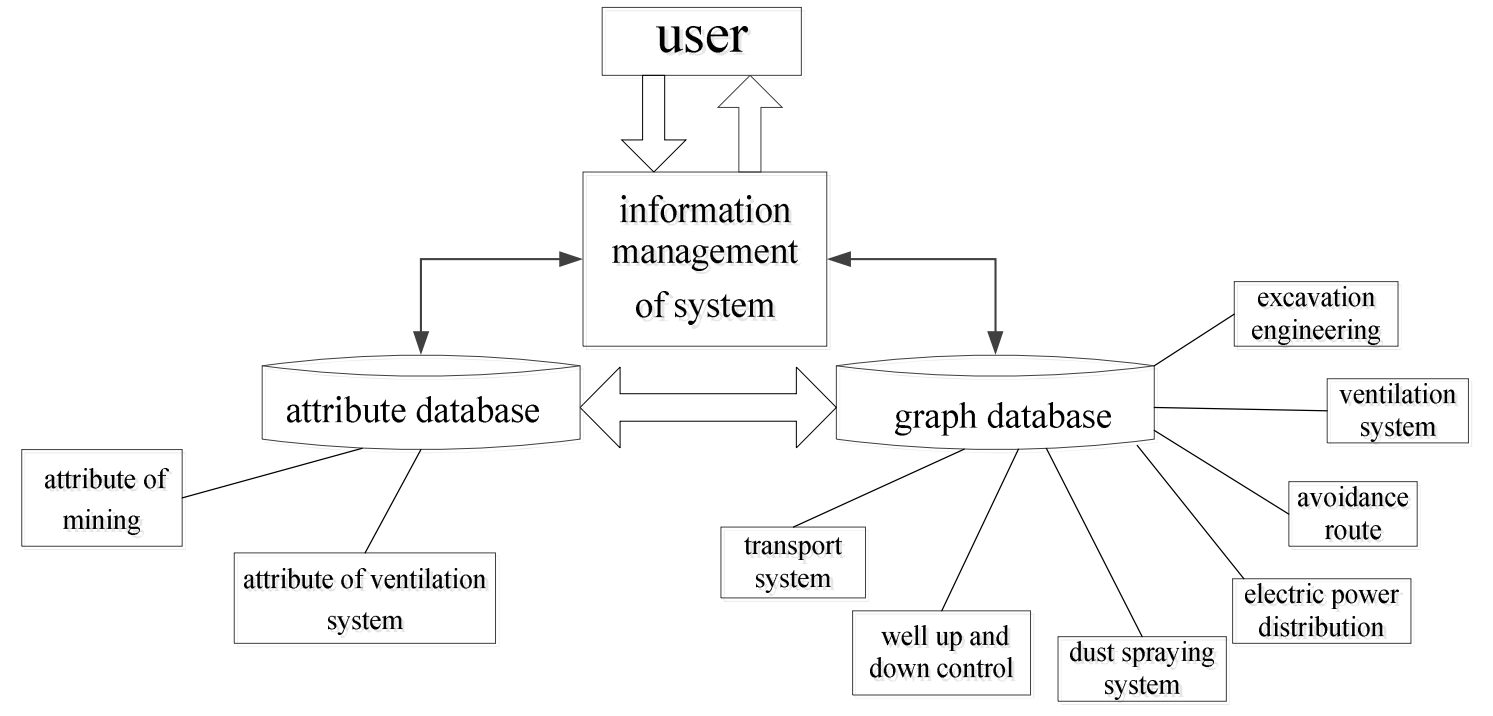

Fig. 3 The database composition and logical relations

Using unified relational database to manage spatial data and attribute data, the model makes the connection between spatial data and attribute data more closely, and tends to be integrated, which can effectively overcome the problem of data inconsistency caused by data update. Server SQL 2008 is used as the database management system, while use spatial data engine of MapInfo to establish the spatial database.

Spatial Database. Spatial database consist of some chart including excavation engineering, ventilation system, avoidance route, electric power distribution, dust spraying system, well up and down control, transport system and thematic layers. Each layer of MapInfo is composed of five files, which include .TAB, .DAT, .IND, .ID, and .MAP as the suffix. Generally, in the same layer, the data have the same set of features and the same attributes.

When the legend of system is created, different numbers are appointed to represent the thematic graphics files in the $6 \mathrm{sub}$ files, including 1 line layer (the basic line of the file), 2 class point layer (basic point file), 3 types of surface layer (basic file), 4 point layer (thematic attribute point file), 5 cable layer (special line properties file) and 6 kinds of surface layer (thematic attribute file).

Attribute Database. When MapInfo storages space data, the system will generate. Map as spatial data files, then the corresponding attribute data is also stored in the. Data file at the same name. In addition, the table structure of attribute data is stored in the Tab file to realize the mutual query of attribute data and spatial data with MapX components. For example: the attribute data of excavation engineering is shown in Table 1 and the attribute data of ventilation system is shown in Table 2 .

Table 1 the attribute data of excavation engineering

\begin{tabular}{cccc}
\hline number & designation & type and length of data & explanation \\
\hline 1 & cj_id & nchar(14) & number of mining face \\
2 & cj_time & datetime & date \\
3 & cj_star & nvarchar(20) & starting point \\
4 & cj_end & nvarchar(20) & terminal point \\
5 & cj_number & int & number of Operators \\
\hline
\end{tabular}


Table 2 the attribute data of ventilation system

\begin{tabular}{cccc}
\hline number & designation & type and length of data & explanation \\
\hline 1 & tf_id & nchar(8) & graphic number \\
2 & tf_device & nchar(14) & number of equipment
\end{tabular}

Engine of Spatial Data Engine. Scheduling data can be achieved in system by using MAPGIS, CAD graphics and other software, then Spatial data engine transform data into MAPGIS format and upload to relational database of SQL Server 2008.In this way, the system can realize data unified management. Its specific process is shown in Fig. 4.

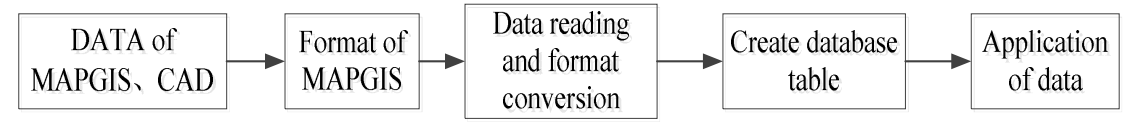

Fig.4 The process of spatial data engine

\section{The Design of WebGIS Based on Ajax and ASP.NET}

In the design of WebGIS based on Ajax and ASP.NET, user interaction by Ajax transmit the parameters of request to the ASP.NET in the server side, then the object in the GIS server is called by the ASP.NET and the desired image is generated[6,7]. Structure of WebGIS based on Ajax and ASP.NET is shown in Fig. 5.

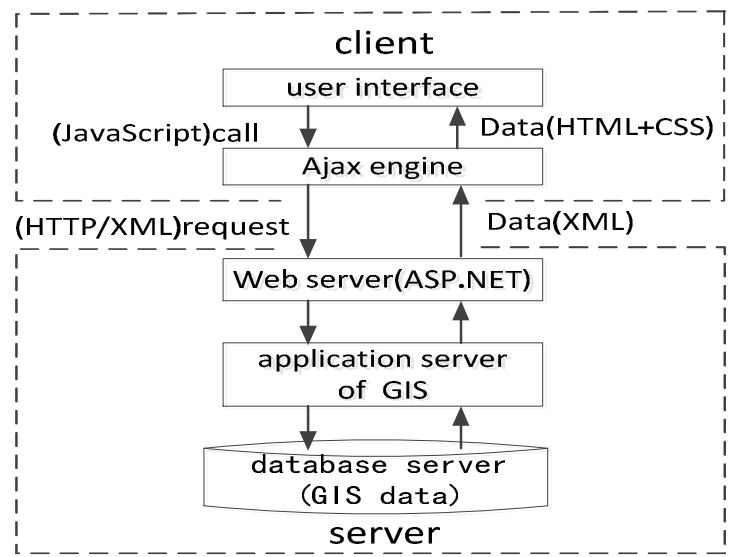

Fig.5 Structure of WebGIS based on Ajax and ASP.NET

At the server side, firstly, the parameters from Ajax are received by ASP.NET. Secondly, the appropriate GIS object is generated according to these parameters. Thirdly, a set of pictures within the geographic range of the request are generated based on these GIS objects. Lastly, this group of pictures is sent to the browser's XMLHttpRequest. In the browser side, the corresponding operation is recorded in the XML string by JavaScript script and sent to the corresponding ASP.NET by XHR. A callback function is defined by XHR, then deal with the results of ASP.NET.

Overview Map Function. Overview map is part of the GIS system essential. Through a display control of map, the system always display map all the time. The program of Overview Map functions is shown in function getOverviewValue().

function getOverviewValue()

\{ var url="MapController.ashx?Command=OverviewValue\&Ran="+ Math.ranDOM(); var mapImage=document.getElementById("MapControl ljmage");

if (mapImage.mapAlias)

url+="\&MapAlias="+maplmage.mapAlias;

var $\mathrm{xmlHttp}=$ CreateXmlHttpQ; // create an instance of the Xm1HttpRequest object 
xmlHttp.open("GET",url,false); // establish a call to the server

xmlHttp.send(null); // send Ajax request

var result=xmlHttp.responseText; \}

return result; // get value

Function of Ajax Image Zoom. The image is processed hierarchically to achieve fast image scaling by early loading and caching. The program of Ajax image zoom is shown in scale(T).

function scale(T)

$\{\quad \operatorname{varT} 1=\mathrm{T} 2=$ sample[0]; // Sample is a scale constant array.

for(var $\mathrm{i}=1 ; \mathrm{i}<$ sample.length; $\mathrm{i}++)$

\{ if(scales $[\mathrm{i}]<\mathrm{T} \& \&$ sample $[\mathrm{i}]>\mathrm{T} 1) \quad \mathrm{T} 1=\mathrm{T}$;

else if(scales $[\mathrm{i}]>\mathrm{T} \& \&$ sample $[\mathrm{i}]<\mathrm{T} 2) \mathrm{T} 2=\mathrm{T}$;

\}

$\mathrm{T} 0=(\mathrm{T} 1+\mathrm{T} 2) / 2$;

return $\mathrm{T}<\mathrm{T} 0$ ? $\mathrm{T} 1: \mathrm{T} 2$;

\}

\section{Conclusion}

Information management information management system of coal mine based on WebGIS include graphic data processing of scheduling information and Graphics browser, which realize the digital management of coal mine information dispatching management and standardize the unified management of scheduling information. In addition, the system improves the utilization of information resources and the level of coal mine information management, and the integration of production, scheduling and planning is realized. Through real-time chart information, the real situation of each coal mine production can be reflected directly. The system can satisfy the request of high yield and high efficiency coal mine modernization construction. With the improvement of remote sensing technology and the global positioning system, through the combination of digital mine construction, highly integrated coal mine geographic information system, can realize the modernization of intelligent management and standardization of coal mine management.

\section{References}

[1] Jian Wang. Design and implementation of coal mine dispatching management information system. University of Electronic Science and technology.2014

[2] Hong Ma. Research and development of WebGIS based scheduling management system. Coal Technology. 2013,03:114-116.

[3] Mingqiang Guo. Research on the key technologies of WebGIS model for high performance computing. China University of Geosciences,2013.

[4] Xiangzhi Xie, Xiucai Guo. Graphic information management system of coal mine dispatching based on GIS Technology. Coal Engineering. 2010,03:109-112.

[5] Yuanxia Tan. Information management system of coal mine production based on WebGIS. Coal Mine Machinery. 2012,08:278-279.

[6] Yanlan Yang, Xiaoxue Jin, Huan Ye. Research on AJAX ASP.NET framework and its application in Web development. Computer Applications and Software. 2011,06:195-198.

[7] James Miller. Refactoring legacy AJAX applications to improve the efficiency of the data exchange component. Journal of Systems and Software, Volume 86, Issue 1, January 2013, Pages $72-88$ 